\title{
Magnetotropic Response in Ruthenium Chloride
}

\author{
I. MANDAL* \\ Institute of Nuclear Physics, Polish Academy of Sciences, \\ W.E. Radzikowskiego 152, 31-342 Kraków, Poland
}

Received: 17.09.2021 \& Accepted: 04.11.2021

Doi: $10.12693 /$ APhysPolA.140.372

*e-mail: ipsita.physics@gmail.com

\begin{abstract}
We consider exchange couplings present in an effective Hamiltonian for $\alpha-\mathrm{RuCl}_{3}$, known as the $\mathrm{K}-\Gamma$ model. The material considered has a honeycomb lattice and is intended to be representative of the Kitaev materials (which can realize the 2D Kitaev model). However, the behaviour of $\mathrm{RuCl}_{3}$ shows that the exchange interactions of this material are not purely Kitaev-like, especially since it has antiferromagnetic order at low temperatures and at low intensities of an applied external magnetic field. Fitting the data obtained from the measurements of the magnetotropic coefficient (thermodynamic coefficient associated with magnetic anisotropy), reported in Nat. Phys. 17, 240 (2021), we estimate the values of the exchange couplings of the effective Hamiltonian. The fits indicate that the Kitaev couplings are subdominant to other exchange couplings.
\end{abstract}

topics: $\alpha-\mathrm{RuCl}_{3}$, magnetotropic coefficient, $\mathrm{K}-\Gamma$ model

\section{Introduction}

Spin-orbit coupling (SOC) assisted $\left(\operatorname{spin} j=\frac{1}{2}\right)$ Mott insulators, showing bond-directional exchange interactions, are expected to exhibit unconventional quantum magnetic phases, such as spin liquids $[1,2]$ predicted by two-dimensional (2D) Kitaev model [3] on a honeycomb lattice. Putative quantum spin liquids are dubbed as "Kitaev spin liquids" (KSLs) [4-8] and materials that exhibit such behaviour are called the Kitaev materials. Compounds like honeycomb iridates and $\alpha-\mathrm{RuCl}_{3}$ have been identified as candidate Kitaev materials. The hallmark feature of the Kitaev material is that the Kitaev coupling is the dominant exchange coupling. However, the behaviour of $\mathrm{RuCl}_{3}$ shows that the exchange interactions of the material are not purely Kitaev-like. In this paper, we address the unresolved question of what possible exchange couplings in $\alpha-\mathrm{RuCl}_{3}[7-11]$ could be, for example, what are the dominant terms in the effective spin Hamiltonian, and whether we can estimate the values of these coupling constants.

At low energies, experiments [12-14] show the signatures consistent with a zig-zag antiferromagnet (AFM) background (also consistent with ab initio calculations $[9,15,16])$, and at the same time indicate the existence of an unconventional quantum magnetic phase, which could be much sought after KSL, induced by a finite magnetic field. Exact numerical diagonalization methods investigating data from dynamical spin structure factors, as well as methods relating to the heat capacity measurements $[17,18]$, found that off-diagonal interactions, not Kitaev interactions, are dominant [19]. In turn, other computational studies $[7,9,10,20]$ report that Kitaev terms are dominant.

We focus on the results from the resonant torsion magnetometry experiment [21] in which the magnetotropic coefficient $k \equiv \frac{\partial^{2} F}{\partial \theta^{2}}$ at temperature $\mathcal{T}$ was measured. Here, $F=-\beta^{-1} \ln (Z)$ is the free energy, $\beta=1 /\left(k_{\mathrm{B}} \mathcal{T}\right)$, and $\theta$ is the angle between the applied magnetic field $\boldsymbol{B}$ and the $c$-axis of the crystal. Using a simple Hamiltonian with a dominant paramagnetic term, we will show that we can fit the data obtained from the measurements of the magnetotropic coefficient, and the fits correspond to the Kitaev terms being subdominant in the socalled $\mathrm{K}-\Gamma$ model.

\section{Model}

Due to the presence of on-site SOC, the effective magnetic field components along the spin projections are given by

$$
\tilde{B}_{\alpha} \equiv B_{\gamma} D_{\gamma \alpha},
$$

where

$$
[D]=\mathcal{A} \mathbb{1}_{3 \times 3}+\left(\begin{array}{ccc}
0 & \mathcal{B} & \mathcal{B} \\
\mathcal{B} & 0 & \mathcal{B} \\
\mathcal{B} & \mathcal{B} & 0
\end{array}\right) .
$$


The form of $[D]$ has been fixed by the $C_{3}$ and $C_{2}$ rotation symmetries [22] of $\mathrm{P} 3_{1} 12$, constraining it to $\mathcal{A}$ and $\mathcal{B}$ as only two independent components (see Appendix A). In the $a b c$-coordinate system, $[D]$ is diagonalized to the form $\operatorname{diag}\left\{g_{a}, g_{a}, g_{c}\right\}$, where the $g$-factors are given by

$$
\begin{aligned}
& \tilde{g}_{a}=\frac{k_{B} g_{a}}{\mu_{\mathrm{B}}}, \quad \tilde{g}_{c}=\frac{k_{B} g_{c}}{\mu_{\mathrm{B}}}, \\
& g_{a}=\mathcal{A}-\mathcal{B}, \quad g_{c}=\mathcal{A}+2 \mathcal{B},
\end{aligned}
$$

such that $k_{\mathrm{B}}=1.38064852 \times 10^{-23} \mathrm{~J} / \mathrm{K}$ and $\mu_{\mathrm{B}}=9.274 \times 10^{-24} \mathrm{~J} / \mathrm{T}$. The SOC forces the leading order paramagnetic term in our model Hamiltonian to be $H_{0}=-\sum_{\alpha=\{x, y, z\}} \tilde{B}_{\alpha} \sigma_{j}^{\alpha}$, rather than $\left(-\sum_{\alpha=\{x, y, z\}} B_{\alpha} \sigma_{j}^{\alpha}\right)$. We notice that the Hamiltonian has the units of $g \mu_{\mathrm{B}} \boldsymbol{\sigma} \cdot \boldsymbol{B}$, where $\boldsymbol{\sigma}$ - the dimensionless spin- $\frac{1}{2}$ vector operator, and $\frac{g \mu_{B} \boldsymbol{\sigma} \cdot \boldsymbol{B}}{k_{\mathrm{B}} \mathcal{T}}$ is dimensionless because of the factors as $\mathrm{e}^{-\beta H}$. Hence, $\mathcal{A}$ and $\mathcal{B}$ have units of $[\mathrm{K} / \mathrm{T}]$.

Following the arguments above, the physics of a honeycomb lattice, restricted to nearest-neighbor interactions and subjected to the external magnetic field $\boldsymbol{B}$, can be captured by a Hamiltonian of the form

$$
H=H_{0}+V
$$

where

$$
H_{0}=-\sum_{\alpha=\{x, y, z\}} \tilde{B}_{\alpha} \sigma_{j}^{\alpha}
$$

and

$$
V=\sum_{\gamma=\{x, y, z\}} \sum_{\langle j k\rangle_{\gamma-\text { links }}} J_{\alpha \beta}^{\gamma} \sigma_{j}^{\alpha} \sigma_{k}^{\beta} .
$$

Now, $H_{0}$ is the leading order part for the large $B$ and $V$ is the subleading part. The second summation (6) runs over nearest-neighbour spins at sites $j$ and $k$, coupled by a bond along the $\gamma=(x, y, z)$ direction. Furthermore, $J_{\alpha \beta}^{\gamma}$ is the coupling constant (for a given value of $\alpha, \beta$, and $\gamma$ ), $\sigma_{j}^{\mu}(\mu=x, y, z)$ is the Pauli spin matrix representing the spin- $\frac{1}{2}$ operator on site $j$, projected along the $\mu$-axis. The spins are located on the vertices of the honeycomb lattice and $\langle j k\rangle$ denotes the labels of the nearest-neighbour spins. For a hexagonal lattice, there are three different kinds of bonds that can be grouped according to their alignments (see Fig. 3 of [3]). These are vertical bonds (which we label as $z$-links), bonds with positive slope (which we label as $x$-links), and bonds with negative slope (which we label as $y$ links). As a results, a given site $j$ is connected to three other sites by these three different types of links, denoted by $\gamma$. In other words, the links are not oriented along the 3D orthogonal Cartesian coordinate directions, but each $\gamma$-value denotes the orientation of the bond we are referring to.

The parameters $J_{\alpha \beta}^{\gamma}$ are obtained from the $\mathrm{K}-\Gamma$ model, where K stands for the Kitaev term of the 2D Kitaev model [3], and $\Gamma$ represents the off-diagonal exchange interactions $[7,8,10,20]$, as follows

$$
\begin{aligned}
H_{K} & =\kappa\left[\sum_{\langle j, k\rangle_{x-\text { links }}} \sigma_{j}^{x} \sigma_{k}^{x}+\sum_{\langle j, k\rangle_{y-\text { links }}} \sigma_{j}^{y} \sigma_{k}^{y}+\sum_{\langle j, k\rangle_{z-\text { links }}} \sigma_{j}^{z} \sigma_{k}^{z}\right], \\
H_{\Gamma} & =\Gamma\left[\sum_{\langle j, k\rangle_{x-\text { links }}}\left(\sigma_{j}^{y} \sigma_{k}^{z}+\sigma_{j}^{z} \sigma_{k}^{y}\right)+\sum_{\langle j, k\rangle_{y-\text { links }}}\left(\sigma_{j}^{z} \sigma_{k}^{x}+\sigma_{j}^{x} \sigma_{k}^{z}\right)+\sum_{\langle j, k\rangle_{z-\text { links }}}\left(\sigma_{j}^{x} \sigma_{k}^{y}+\sigma_{j}^{y} \sigma_{k}^{x}\right)\right] \\
+ & {\left[\sum_{\langle j, k\rangle_{x-\text { links }}}\left(\sigma_{j}^{y} \sigma_{k}^{z}-\sigma_{j}^{z} \sigma_{k}^{y}\right)+\sum_{\langle j, k\rangle_{y-\text { links }}}\left(\sigma_{j}^{z} \sigma_{k}^{x}-\sigma_{j}^{x} \sigma_{k}^{z}\right)+\sum_{\langle j, k\rangle_{z-\text { links }}}\left(\sigma_{j}^{x} \sigma_{k}^{y}-\sigma_{j}^{y} \sigma_{k}^{x}\right)\right] . }
\end{aligned}
$$

Here, $\kappa$ is the strength of the Kitaev term, and $\Gamma$ represents the off-diagonal exchange interaction strengths. The parameters $\Gamma$ and $\mathcal{D}$ represent, respectively, the symmetric and antisymmetric parts of the off-diagonal terms.

If the trivial paramagnetic term $H_{0}$ dominates the system's response to the external magnetic field, we can perform a thermodynamic expansion of the partition function [23], as reviewed in Appendix B. Then, the partition function corrected to leading order evaluates to

$$
\begin{aligned}
\mathcal{Z}(T) & =(2 \cosh (\beta \tilde{B}))^{2 N_{c}} \\
\times & {\left[1+\beta N_{c} \sum_{\gamma, \alpha^{\prime}, \lambda^{\prime}} J_{\alpha^{\prime} \lambda^{\prime}}^{\gamma} \frac{\sinh \left(\beta \tilde{B}_{\alpha^{\prime}}\right) \sinh \left(\beta \tilde{B}_{\lambda^{\prime}}\right)}{\cosh ^{2}(\beta \tilde{B})}\right], }
\end{aligned}
$$

where

$$
\tilde{B}=\sqrt{\sum_{\alpha} \tilde{B}_{\alpha} \tilde{B}_{\alpha}}
$$

and $N_{c}$ is the number of unit cells (or half the number of honeycomb lattice sites) in the system. It turns out that this simple model can indeed explain the experimental data to a high degree of precision.

\section{Fitting the data}

According to some papers in the literature [9, 24], the point-group symmetry of the $\mathrm{Ru}-\mathrm{Ru}$ links is $C_{2 h}$ in the $C / 2 m$ unit cell, and hence the antisymmetric Dzyaloshinskii-Moriya (DM) exchange is zero. Because spin is an axial vector, the non-zero antisymmetric part of $J_{\alpha \beta}^{\gamma}$ is equivalent to $\boldsymbol{P} \cdot(\boldsymbol{S} \times \boldsymbol{S})$ term, where $\boldsymbol{P}$ is the polar vector. Therefore, to 


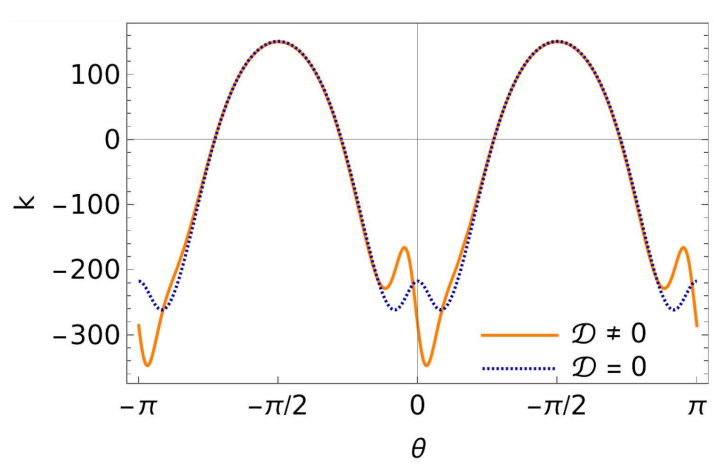

Fig. 1. Results of $k$ versus $\theta$ computed from our theoretical model, where the orange curve represents the one obtained with the best-fit parameters for the $(\mathcal{T}=20 \mathrm{~K}, B=30 \mathrm{~T})$ data set, whereas the dotted red curve has been drawn using those same parameters, except that we have set $\mathcal{D}=0$. This clearly shows that we can never get an asymmetric spike around $\theta=0$ without an asymmetric offdiagonal $\Gamma$ term, which represents DM interactions.

have a DM term in addition, the chemical environment of the $\mathrm{Ru}-\mathrm{Ru}$ bond must include a polar vector. In the undistorted honeycomb lattice, a polar vector is prohibited by the symmetry. It is non-zero for the next-nearest neighbor exchange links (even in the undistorted case) [25] or if $\mathrm{Cl}$ octahedra are distorted.

However, the peak shapes in the $k$ versus $\theta$ data (and the behavior in a broader angular range around it) are not symmetric around the $c$-axis, i.e., $k(\theta)-k(-\theta) \neq 0$. We have found that this behavior is only possible if the antisymmetric DM term is taken into account, which points to distorted octahedra, leading to a deviation from the assumed crystal symmetries. This is shown in Fig. 1, where are the $k(\theta)$ results computed from our model, with the parameter values taken from the best-fit parameters of the $B=30 \mathrm{~T}$ data (except that we set $\mathcal{D}=0$ for the orange curve).

The expressions for $F$ and $k$ depend on the polar angle $\theta$ but not on the azimuthal angle $\phi$. We fit the data sets for four different values of the applied magnetic field strength $B$, using "NonlinearModelFit" of Mathematica. The data sets for $B \leq 15 \mathrm{~T}$ are not considered as they are either close to, or within the AFM phase. Since the scaling and absolute shift of each data set are uncertain, we include two more parameters, namely " $\zeta$ " and " $\eta$ ", corresponding to the unknown scale and shift, respectively. The experimental data and the fitted functions are shown in Fig. 2. The confidence intervals for all the parameters at $67 \%$ confidence level are shown in Table I.

We also fit the $k$ versus $B$ data available for $\theta=\pi / 2$. It can be checked that the correction terms from $J_{\alpha \beta}^{\gamma}$ hardly affect the regions around $\theta=\pi / 2$ (see Fig. 3). The most visible impact of these correction terms occurs only around $\theta=0$ and $\theta=\pi$ regions. Hence, the fitting process keeping the first-order correction makes the parameters indeterminate. However, if we fit only the zerothorder expression, we get excellent values for $g_{a}$ and $g_{c}$. We also need to include a parameter $\eta$ to account for the uncertainty in the absolute shift of the data set for each temperature value. The fit results are shown in Fig. 4. The data sets for
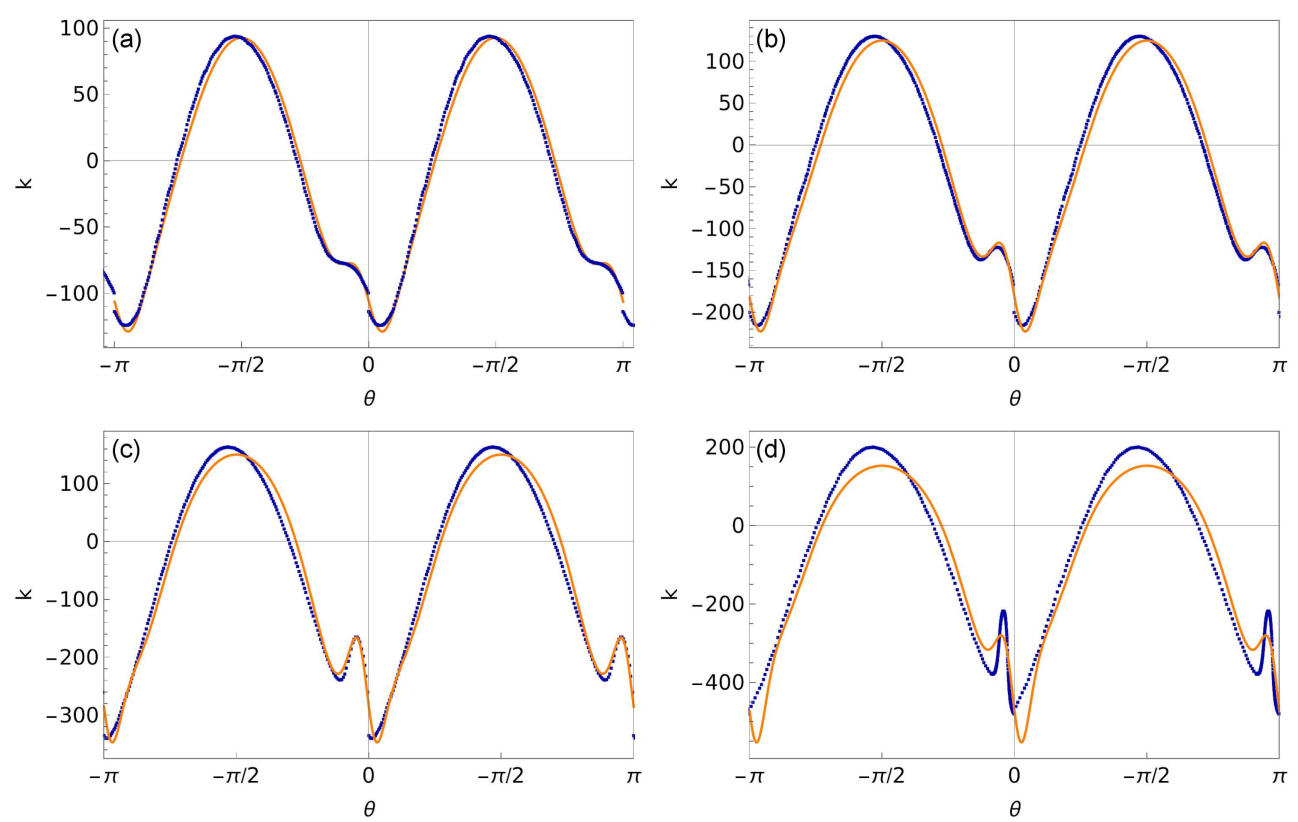

Fig. 2. The data sets for $k$ versus $\theta$ (in radians) at $\mathcal{T}=20 \mathrm{~K}$ for various values of the applied magnetic field strength $B$ [T]: (a) $B=20 \mathrm{~T}$, (b) $B=25 \mathrm{~T}$, (c) $B=30 \mathrm{~T}$, (d) $B=34.5 \mathrm{~T}$. We have represented the experimental data-points in red, and the best-fit curves in orange. 
TABLE I

TABLE II

The fitting of parameters at $67 \%$ confidence level for the $k$ versus $\theta$ data set. Here, $B$ and $\kappa, \Gamma, \mathcal{D}$ are in units of $[\mathrm{T}], g_{a}, g_{c}$ are in units of $[\mathrm{K} / \mathrm{T}], \zeta$ is dimensionless, and $\eta$ has the same unit as $k$.

\begin{tabular}{|c|c|c|c|}
\hline & Estimate & Std. error & Confidence interval \\
\hline \multicolumn{4}{|c|}{$B=20 \mathrm{~T}$} \\
\hline$\kappa$ & 54.4 & 2.37 & $(52.1,56.7)$ \\
\hline$\Gamma$ & 102 & 6.09 & $(96.8,108)$ \\
\hline $\mathcal{D}$ & -9.60 & 0.629 & $(-10.2,-8.98)$ \\
\hline$g_{a}$ & 4.00 & 0.137 & $(3.87,4.13)$ \\
\hline$g_{c}$ & 1.79 & 0.0984 & $(1.70,1.89)$ \\
\hline$\zeta$ & 2.00 & 0.0736 & $(1.93,2.07)$ \\
\hline$\eta$ & -9.38 & 0.150 & $(-9.53,-9.234)$ \\
\hline \multicolumn{4}{|c|}{$B=25 \mathrm{~T}$} \\
\hline$\kappa$ & 74.6 & 1.32 & $(73.3,75.9)$ \\
\hline$\Gamma$ & 133 & 8.80 & $(125,142)$ \\
\hline $\mathcal{D}$ & -13.9 & 0.866 & $(-14.7,-13.1)$ \\
\hline$g_{a}$ & 4.00 & 0.124 & $(3.88,4.12)$ \\
\hline$g_{c}$ & 1.52 & 0.0677 & $(1.46,1.59)$ \\
\hline$\zeta$ & 2.00 & 0.071 & $(1.93,2.07)$ \\
\hline$\eta$ & -24.4 & 0.281 & $(-24.7,-24.2)$ \\
\hline \multicolumn{4}{|c|}{$B=30 \mathrm{~T}$} \\
\hline$\kappa$ & 95.8 & 1.46 & $(94.4,97.3)$ \\
\hline$\Gamma$ & 152 & 9.46 & $(143,161)$ \\
\hline $\mathcal{D}$ & -15.8 & 0.841 & $(-16.6,-15.0)$ \\
\hline$g_{a}$ & 4.00 & 0.118 & $(3.88,4.11)$ \\
\hline$g_{c}$ & 1.26 & 0.0486 & $(1.21,1.30)$ \\
\hline$\zeta$ & 2.00 & 0.0687 & $(1.93,2.07)$ \\
\hline$\eta$ & -50.1 & 0.507 & $(-50.6,-49.6)$ \\
\hline \multicolumn{4}{|c|}{$B=34.5 \mathrm{~T}$} \\
\hline$\kappa$ & 85.5 & 2.90 & $(82.6,88.3)$ \\
\hline$\Gamma$ & 156 & 28.7 & $(128,184)$ \\
\hline $\mathcal{D}$ & -20.3 & 3.31 & $(-23.5,-17.1)$ \\
\hline$g_{a}$ & 4.00 & 0.276 & $(3.73,4.27)$ \\
\hline$g_{c}$ & 1.20 & 0.122 & $(1.08,1.32)$ \\
\hline$\zeta$ & 2.00 & 0.145 & $(1.86,2.14)$ \\
\hline$\eta$ & -88.8 & 1.42 & $(-90.2,-87.4)$ \\
\hline
\end{tabular}

temperatures $\mathcal{T} \leq 20 \mathrm{~K}$ are not considered since each of them has a considerable region within the AFM phase in the low $B$ ranges, which cannot be fitted by the functional forms meant for the paramagnetic phase. The confidence intervals for $g_{a}, g_{c}$, and $\eta$, at $67 \%$ confidence level, are shown in Table II.

\section{Summary and outlook}

Let us discuss some other possibilities which might be responsible for causing the asymmetry in the spike around $\theta=0$ in the $k$ versus $\theta$ data. Firstly, in the experimental setups, the path along which the sample is rotated in the external magnetic field to change $\theta$ may deviate from a great circle, leading to an uncertainty of up to $10^{\circ}$.
The fitting of parameters at $67 \%$ confidence level for the $k$ versus $B$ data set. Here, temperature $\mathcal{T}$ is in units $[\mathrm{K}], g_{a}, g_{c}$ are in units of $[\mathrm{K} / \mathrm{T}]$, and $\eta$ has the same unit as $k$.

\begin{tabular}{|c|c|c|c|}
\hline & Estimate & Std. error & Confidence interval \\
\hline \multicolumn{4}{|c|}{$\mathcal{T}=30 \mathrm{~K}$} \\
\hline$g_{a}$ & 2.43 & 0.00172 & $(2.43,2.44)$ \\
\hline$g_{c}$ & 1.20 & 0.00148 & $(1.2,1.20)$ \\
\hline$\eta$ & 0.000131 & 0.0158 & $(-0.0152,0.0155)$ \\
\hline \multicolumn{4}{|c|}{$\mathcal{T}=40 \mathrm{~K}$} \\
\hline$g_{a}$ & 2.46 & 0.000785 & $(2.46,2.46)$ \\
\hline$g_{c}$ & 1.24 & 0.000738 & $(1.24,1.24)$ \\
\hline$\eta$ & 0.000267 & 0.00700 & $(-0.00655,0.00709)$ \\
\hline \multicolumn{4}{|c|}{$\mathcal{T}=50 \mathrm{~K}$} \\
\hline$g_{a}$ & 2.42 & 0.000581 & $(2.42,2.42)$ \\
\hline$g_{c}$ & 1.23 & 0.000600 & $(1.23,1.23)$ \\
\hline$\eta$ & 0.00822 & 0.00473 & $(0.00362,0.0128)$ \\
\hline \multicolumn{4}{|c|}{$\mathcal{T}=60 \mathrm{~K}$} \\
\hline$g_{a}$ & 2.34 & 0.000456 & $(2.34,2.34)$ \\
\hline$g_{c}$ & 1.20 & 0.000518 & $(1.20,1.20)$ \\
\hline$\eta$ & 0.00592 & 0.00310 & $(0.00290,0.00894)$ \\
\hline \multicolumn{4}{|c|}{$\mathcal{T}=70 \mathrm{~K}$} \\
\hline$g_{a}$ & 2.28 & 0.000920 & $(2.28,2.28)$ \\
\hline$g_{c}$ & 1.20 & 0.00113 & $(1.20,1.20)$ \\
\hline$\eta$ & 0.00923 & 0.00490 & $(0.00446,0.0140)$ \\
\hline \multicolumn{4}{|c|}{$\mathcal{T}=80 \mathrm{~K}$} \\
\hline$g_{a}$ & 2.20 & 0.00158 & $(2.2,2.2)$ \\
\hline$g_{c}$ & 1.20 & 0.00204 & $(1.20,1.20)$ \\
\hline$\eta$ & 0.0452 & 0.00637 & $(0.039,0.0514)$ \\
\hline \multicolumn{4}{|c|}{$\mathcal{T}=90 \mathrm{~K}$} \\
\hline$g_{a}$ & 2.12 & 0.00235 & $(2.12,2.12)$ \\
\hline$g_{c}$ & 1.20 & 0.00316 & $(1.20,1.20)$ \\
\hline$\eta$ & -0.0124 & 0.00691 & $(-0.0191,-0.00565)$ \\
\hline \multicolumn{4}{|c|}{$\mathcal{T}=100 \mathrm{~K}$} \\
\hline$g_{a}$ & 2.03 & 0.00351 & $(2.03,2.03)$ \\
\hline$g_{c}$ & 1.20 & 0.00481 & $(1.20,1.20)$ \\
\hline$\eta$ & 0.043 & 0.00740 & $(0.0358,0.0503)$ \\
\hline \multicolumn{4}{|c|}{$\mathcal{T}=110 \mathrm{~K}$} \\
\hline$g_{a}$ & 1.94 & 0.00531 & $(1.94,1.95)$ \\
\hline$g_{c}$ & 1.20 & 0.00732 & $(1.19,1.201)$ \\
\hline$\eta$ & -0.00637 & 0.0078 & $(-0.014,0.00123)$ \\
\hline \multicolumn{4}{|c|}{$\mathcal{T}=130 \mathrm{~K}$} \\
\hline$g_{a}$ & 1.79 & 0.0111 & $(1.78,1.80)$ \\
\hline$g_{c}$ & 1.20 & 0.0151 & $(1.18,1.21)$ \\
\hline$\eta$ & -0.0081 & 0.00815 & $(-0.016,-0.000166)$ \\
\hline \multicolumn{4}{|c|}{$\mathcal{T}=150 \mathrm{~K}$} \\
\hline$g_{a}$ & 1.76 & 0.0953 & $(1.66,1.85)$ \\
\hline$g_{c}$ & 1.287 & 0.122 & $(1.17,1.41)$ \\
\hline$\eta$ & -0.00276 & 0.0385 & $(-0.0403,0.0347)$ \\
\hline
\end{tabular}




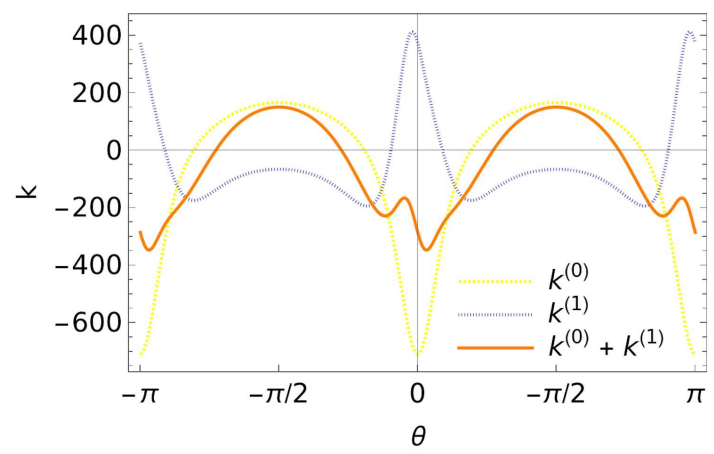

Fig. 3. Three curves corresponding to leading order expression $k^{(0)}$ (yellow), first order correction $k^{(1)}$ (blue), and $k^{(0)}+k^{(1)}$ (orange), as functions of $\theta$. The results have been computed from our theoretical model, using the best-fit parameters for the $(\mathcal{T}=20 \mathrm{~K}, B=30 \mathrm{~T})$ data set. These three curves are shown in dashed yellow, dotted red, and orange, respectively.

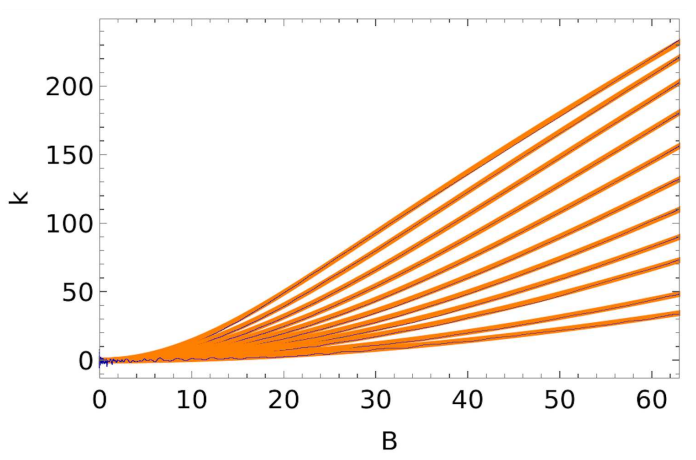

Fig. 4. The data sets for $k$ versus $B$ (in units of [T]) at $\theta=\pi / 2$ for temperatures ranging from $\mathcal{T}=30 \mathrm{~K}$ to $\mathcal{T}=150 \mathrm{~K}$ at intervals of $10 \mathrm{~K}$. The topmost curve represents the $30 \mathrm{~K}$ data, whereas the lowermost curve represents the $150 \mathrm{~K}$ data. We have represented the experimental data-points in red, and the best-fit curves in orange.

However, incorporating these deviations, the theoretical curves do not show the desired asymmetry. Secondly, the K- $\Gamma$ Hamiltonian (even without DM, distortion, misalignment of rotation, etc.) lacks mirror reflection symmetry. Therefore, magnetotropic coefficients (or free energy) are different for applied magnetic fields $\boldsymbol{B}$ and $\boldsymbol{B}^{\prime}$ that are related by a mirror reflection in the honeycomb plane. Such asymmetry is artificially removed in the low-order perturbation theory. This is directly analogous to acci- dental symmetries of the standard model (such as separate conservation of baryon ad lepton number) that only exist in the lowest order of expansion in the inverse GUT scale. It is a general phenomenon - low orders in perturbation theory tend to accidentally "restore" some of the symmetries of the Hamiltonian. In the Kitaev model, the lowest order perturbation expansion in magnetic field [3] is symmetric (with respect to mirror- $a b$-plane). To see the asymmetry of the Hamiltonian, one needs to go to higher orders in $B$. The same might be true for the thermodynamic perturbative expansion. Going to higher orders (second, or maybe third order), the asymmetric character of the Hamiltonian may eventually show up. However, such higher order computations are beyond the scope of this paper.

Our best-fit parameters indicate that the Kitaev terms are subdominant to the $\Gamma$ (and $\mathcal{D}$ ) terms. In fact, the large $\Gamma$ value contrasts with the expectation so far that $\alpha-\mathrm{RuCl}_{3}$ is a "Kitaev model material" $[7,9,10,20]$. It has also been predicted in those models (including a small Heisenberg term) $[9,20,26]$ that the ratio $g_{c} / g_{a} \simeq 0.4-0.5$. Our results (see Fig. 1) are close to the aforementioned results, although we should remember that our model differs from the those mentioned.

\section{Acknowledgments}

We thank Michael J. Lawler for suggesting the problem. We are also grateful to Brad Ramshaw, Arkady Shekhter, and Kimberly Modic for insightful discussions.

\section{Appendix A: Choice of coordinate system and crystal symmetries}

We choose a coordinate system such that the plane of the honeycomb lattice is described by three in-plane vectors $\boldsymbol{r}_{1}=(0,1,-1), \boldsymbol{r}_{2}=(-1,1,0)$, and $\boldsymbol{r}_{3}=(-1,0,1)$. These vectors lie on the plane formed by cutting the three points $(1,0,0),(0,1,0)$, and $(0,0,1)$. Then, the perpendicular vector is $\boldsymbol{r}_{\perp}=\frac{1}{\sqrt{3}}(1,1,1)$ and we choose the in-plane direction as $\frac{1}{\sqrt{2}} \boldsymbol{r}_{2}$, giving $\boldsymbol{B} / B=\frac{1}{\sqrt{3}}(1,1,1) \cos (\theta)+$ $\frac{1}{\sqrt{2}}(-1,1,0) \sin (\theta)$, with the magnetic field making an angle $\theta$ with the $c$-axis.

Let us also define the $a$-axis along the line joining $\frac{1}{3}(1,1,1)$ and $(1,0,0)$, such that the projection of $\boldsymbol{B}$ on the $a b$-plane makes an angle $\phi$ with the $a$-axis.

Given a unit vector $\boldsymbol{u}$, the matrix for a rotation by an angle of $\phi$ about an axis in the direction of $\boldsymbol{u}$ is

$R(\boldsymbol{u}, \phi)=\left(\begin{array}{ccc}\cos (\phi)+u_{x}^{2}(1-\cos (\phi)) & u_{x} u_{y}(1-\cos (\phi))-u_{z} \sin (\phi) & u_{x} u_{z}(1-\cos (\phi))+u_{y} \sin (\phi) \\ u_{y} u_{x}(1-\cos (\phi))+u_{z} \sin (\phi) & \cos (\phi)+u_{y}^{2}(1-\cos (\phi)) & u_{y} u_{z}(1-\cos (\phi))-u_{x} \sin (\phi) \\ u_{z} u_{x}(1-\cos (\phi))-u_{y} \sin (\phi) & u_{z} u_{y}(1-\cos (\phi))+u_{x} \sin (\phi) & \cos (\phi)+u_{x}^{2}(1-\cos (\phi))\end{array}\right)$ 
Now the crystal symmetry allows invariance under a $C_{3}$ rotation about $\boldsymbol{r}_{\perp}$, which corresponds to invariance under the rotation matrix

$$
R\left(\boldsymbol{r}_{\perp}, \frac{2 \pi}{3}\right)=\left(\begin{array}{lll}
0 & 0 & 1 \\
1 & 0 & 0 \\
0 & 1 & 0
\end{array}\right) .
$$

For $C_{2}$ rotation about $\boldsymbol{r}_{1}$, we have

$$
R\left(\frac{\boldsymbol{r}_{1}}{\sqrt{2}}, \pi\right)=\left(\begin{array}{ccc}
-1 & 0 & 0 \\
0 & 0 & -1 \\
0 & -1 & 0
\end{array}\right)
$$

Due to on-site spin-orbit coupling, the leading order paramagnetic term in our model is given by

$$
H_{0}=-\sum_{\alpha=\{x, y, z\}} \tilde{B}_{\alpha} \sigma_{j}^{\alpha},
$$

rather than

$$
\left(-\sum_{\alpha=\{x, y, z\}} B_{\alpha} \sigma_{j}^{\alpha}\right), \text { where } \tilde{B}_{\alpha} \equiv B_{\gamma} D_{\gamma \alpha} .
$$

We still have the $C_{3}$ and $C_{2}$ rotation symmetries of $\mathrm{P} 3_{1} 12[22]$ to be satisfied, which implies that

$$
[B]^{\mathrm{T}}[D][\sigma]=(R[B])^{\mathrm{T}}[D] R[\sigma] \Rightarrow D=R^{\mathrm{T}}[D] R,
$$

where $R$ has been defined in (12). Then, $R\left(\boldsymbol{r}_{\perp}, 2 \pi / 3\right)$ and $R\left(\boldsymbol{r}_{1} / \sqrt{2}, \pi\right)$ restrict $[D]$ to have only two independent components, namely $\mathcal{A}$ and $\mathcal{B}$. Therefore,

$$
[D]=\mathcal{A} \mathbb{1}_{3 \times 3}+\left(\begin{array}{ccc}
0 & \mathcal{B} & \mathcal{B} \\
\mathcal{B} & 0 & \mathcal{B} \\
\mathcal{B} & \mathcal{B} & 0
\end{array}\right)
$$

\section{Appendix B: Thermodynamic expansion of the $\mathrm{K}-\Gamma$ model in the large magnetic field limit}

We perform a thermodynamic expansion of the $\mathrm{K}-\Gamma$ model in the large magnetic field limit, following the methods describe in [23], which are applicable when we are interested in the thermodynamic properties at finite temperature. We review this perturbation expansion when the Hamiltonian can be written as $H=H_{0}+\lambda V$, where $H_{0}$ is the leading order part for large $B$, and $\lambda$ is the perturbative expansion parameter, with $V$ being the subleading part.

We are interested in the thermodynamic properties at finite temperature. Thus we start with the canonical partition function

$$
\mathcal{Z}(T)=\operatorname{Tr}\left(\mathrm{e}^{-\beta H}\right)=\operatorname{Tr}\left[\mathrm{e}^{-\beta\left(H_{0}+\lambda V\right)}\right)
$$

and seek to expand its logarithm in powers of $\lambda$. Since $H_{0}$ and $V$ do not commute for the K- $\Gamma$ model, we use the approach employed for interaction picture time evolution. We define the function $f(\beta)$ in the following way

$$
\begin{aligned}
& \mathrm{e}^{-\beta\left(H_{0}+\lambda V\right)}=\mathrm{e}^{-\beta H_{0}} f(\beta) \Rightarrow \\
& \quad \frac{\mathrm{d} f(\beta)}{\mathrm{d} \beta}=-\lambda \mathrm{e}^{\beta H_{0}} V \mathrm{e}^{-\beta H_{0}} f(\beta) .
\end{aligned}
$$

Casting this in the form of the integral equation, we get

$$
f(\beta)=1-\lambda \int_{0}^{\beta} \mathrm{d} \tau \tilde{V}(\tau) f(\tau)
$$

where

$$
\tilde{V}(\tau)=\mathrm{e}^{\tau H_{0}} V \mathrm{e}^{-\tau H_{0}} .
$$

We solve the above by iteration

$$
\begin{gathered}
f(\beta)=1+\sum_{n=1}^{\infty}(-\lambda)^{n} \int_{0}^{\beta} \mathrm{d} \tau_{1} \int_{0}^{\tau_{1}} \mathrm{~d} \tau_{2} \ldots \\
\int_{0}^{\tau_{n-1}} \mathrm{~d} \tau_{n} \tilde{V}\left(\tau_{1}\right) \tilde{V}\left(\tau_{2}\right) \ldots \tilde{V}\left(\tau_{n}\right) .
\end{gathered}
$$

This gives us the partition function as

$$
\begin{gathered}
\mathcal{Z}(T)=\mathcal{Z}_{0}\left[1+\sum_{n=1}^{\infty}(-\lambda)^{n} \int_{0}^{\beta} \mathrm{d} \tau_{1} \int_{0}^{\tau_{1}} \mathrm{~d} \tau_{2} \ldots\right. \\
\left.\int_{0}^{\tau_{n-1}} \mathrm{~d} \tau_{n}\left\langle\tilde{V}\left(\tau_{1}\right) \tilde{V}\left(\tau_{2}\right) \ldots \tilde{V}\left(\tau_{n}\right)\right\rangle_{0}\right]
\end{gathered}
$$

where $\langle\ldots\rangle_{0}$ denotes the unperturbed expectation value

$$
\langle A\rangle_{0} \equiv \frac{\operatorname{Tr}\left(\mathrm{e}^{-\beta H_{0}} A\right)}{\operatorname{Tr}\left(\mathrm{e}^{-\beta H_{0}}\right)}
$$

for any operator $A$. The leading order term is given by

$$
\begin{aligned}
& \langle\tilde{V}(\tau)\rangle_{0}=\frac{\operatorname{Tr}\left(\mathrm{e}^{-\beta H_{0}} \mathrm{e}^{\tau H_{0}} V \mathrm{e}^{-\tau H_{0}}\right)}{\operatorname{Tr}\left(\mathrm{e}^{-\beta H_{0}}\right)}= \\
& \quad \frac{\operatorname{Tr}\left(\mathrm{e}^{-\beta H_{0}} V\right)}{\operatorname{Tr}\left(\mathrm{e}^{-\beta H_{0}}\right)},
\end{aligned}
$$

which is in fact independent of $\tau$.

Let us compute the leading term in the partition function for the Hamiltonian of the main text, such that

$$
\begin{aligned}
& H_{0}=-\sum_{\alpha=\{x, y, z\}} \tilde{B}_{\alpha} \sigma_{j}^{\alpha}, \\
& V=\sum_{\gamma} \sum_{\langle j k\rangle_{\gamma-\text { links }}} J_{\alpha \beta}^{\gamma} \sigma_{j}^{\alpha} \sigma_{k}^{\beta} .
\end{aligned}
$$

Hence, we obtain

$$
\langle\tilde{V}(\tau)\rangle_{0}=\frac{\sum_{\gamma, \alpha^{\prime}, \lambda^{\prime}} \sum_{\langle j l\rangle_{\gamma-\text { links }}} J_{\alpha^{\prime} \lambda^{\prime}}^{\gamma} \sinh \left(\beta \tilde{B}_{\alpha^{\prime}}\right) \sinh \left(\beta \tilde{B}_{\lambda^{\prime}}\right)}{\cosh ^{2}(\beta \tilde{B})}=\frac{N_{c} \sum_{\gamma, \alpha^{\prime}, \lambda^{\prime}} J_{\alpha^{\prime} \lambda^{\prime}}^{\gamma} \sinh \left(\beta \tilde{B}_{\alpha^{\prime}}\right) \sinh \left(\beta \tilde{B}_{\lambda^{\prime}}\right)}{\cosh ^{2}(\beta \tilde{B})},
$$


where $\tilde{B}=\sqrt{\sum_{\alpha} \tilde{B}_{\alpha} \tilde{B}_{\alpha}}$, and $N_{c}$ is the number of unit cells in the system. Finally, this gives us the partition function, corrected to leading order, as

$$
\begin{aligned}
\mathcal{Z}(T) & =[2 \cosh (\beta \tilde{B})]^{2 N_{c}} \\
\times & {\left[1+\beta N_{c} \sum_{\gamma, \alpha^{\prime}, \lambda^{\prime}} J_{\alpha^{\prime} \lambda^{\prime}}^{\gamma} \frac{\sinh \left(\beta \tilde{B}_{\alpha^{\prime}}\right) \sinh \left(\beta \tilde{B}_{\lambda^{\prime}}\right)}{\cosh ^{2}(\beta \tilde{B})}\right] . }
\end{aligned}
$$

\section{References}

[1] L. Balents, Nature 464, 199 (2010).

[2] L. Savary, L. Balents, Rep. Prog. Phys. 80, 016502 (2017).

[3] A. Kitaev, Ann. Phys. 321, 2 (2006).

[4] G. Jackeli, G. Khaliullin, Phys. Rev. Lett. 102, 017205 (2009).

[5] J.G. Rau, E.K.-H. Lee, H.-Y. Kee, Phys. Rev. Lett. 112, 077204 (2014).

[6] K.W. Plumb, J.P. Clancy, L.J. Sandilands, V.V. Shankar, Y.F. Hu, K.S. Burch, H.-Y. Kee, Y.-J. Kim, Phys. Rev. B 90, 041112 (2014).

[7] S.M. Winter, Y. Li, H.O. Jeschke, R. Valentí, Phys. Rev. B 93, 214431 (2016).

[8] S. Trebst, arXiv:1701.07056, 2017.

[9] R. Yadav, N.A. Bogdanov, V.M. Katukuri, S. Nishimoto, J. van den Brink, L. Hozoi, Sci. Rep. 6, 37925 (2016).

[10] Y. Sizyuk, P. Wölfle, N.B. Perkins, Phys. Rev. B 94, 085109 (2016).

[11] K. Riedl, Ying Li, S.M. Winter, R. Valentí, Phys. Rev. Lett. 122, 197202 (2019).

[12] A. Banerjee, C.A. Bridges, J.Q. Yan et al., Nature Mater. 15, 733 (2016).

[13] A. Banerjee, P. Lampen-Kelley, J. Knolle et al., npj Quantum Mater. 3, 8 (2018).
[14] F. Lang, P.J. Baker, A.A. Haghighirad, Y. Li, D. Prabhakaran, R. Valentí, S.J. Blundell, Phys. Rev. B 94, 020407 (2016).

[15] R.D. Johnson, S.C. Williams, A.A. Haghighirad et al., Phys. Rev. B 92, 235119 (2015).

[16] H.-S. Kim, Vijay Shankar V, A. Catuneanu, H.-Y. Kee, Phys. Rev. B 91, 241110 (2015).

[17] S.-H. Do, S.-Y. Park, J. Yoshitake et al., Nature Phys. 13, 1079 (2017).

[18] I.A. Leahy, C.A. Pocs, P.E. Siegfried, D. Graf, S.H. Do, K.Y. Choi, B. Normand, M. Lee, Phys. Rev. Lett. 118, 187203 (2017).

[19] T. Suzuki, S. Suga, Phys. Rev. B 97, 134424 (2018).

[20] J.C.V. Chaloupka, G. Khaliullin, Phys. Rev. B 94, 064435 (2016).

[21] K.A. Modic, R.D. McDonald, J.P.C. Ruff et al., Nature Phys. 17, 240 (2020).

[22] H.-S. Kim, H.-Y. Kee, Phys. Rev. B 93 , 155143 (2016).

[23] J. Oitmaa, C. Hamer, W. Zheng, Series Expansion Methods for Strongly Interacting Lattice Models, Cambridge University Press, Cambridge 2006.

[24] H.B. Cao, A. Banerjee, J.Q. Yan, C.A. Bridges, M.D. Lumsden, D.G. Mandrus, D.A. Tennant, B.C. Chakoumakos, S.E. Nagler, Phys. Rev. B 93, 134423 (2016).

[25] F.D.M. Haldane, Phys. Rev. Lett. 61, 2015 (1988).

[26] M. Majumder, M. Schmidt, H. Rosner, A.A. Tsirlin, H. Yasuoka, M. Baenitz, Phys. Rev. B 91, 180401 (2015). 